\title{
Apparatus for auditory stimulus sorting
}

\section{P. D. BRICKER, S. C. JOHNSON, and C. F. MATTKE, BELL TELEPHONE LABORATORIES, Murray Hill, New Jersey 07974}

An apparatus that enables Ss to sort and rank auditory stimuli is described. It consists of an array of movable pushbuttons that may be connected to various stimulus generators.

Sorting and ranking tasks, often useful in the study of certain perceptual relations, are best used with stimuli that Ss can manipulate. Many visual, tactual, and kinesthetic stimuli are manipulable, but auditory stimuli are not generally thought to be so. For this reason, studies of auditory perception that might otherwise have used sorting or ranking tasks have typically used the method of paired comparisons, which becomes extremely tedious as the number of stimuli increases.

The apparatus described here allows Ss to manipulate any auditory stimulus that can be produced by pressing an electrical pushbutton. The device consists of an array of movable pushbuttons, each of which is a visual symbol for the auditory stimulus that is produced by pressing that button. By moving the buttons, Ss can form ordinal, categorical, or spatial arrangements of the auditory stimuli, according to E's instructions. For example, buttons representing auditory stimuli may be placed in vertical arrays for sorting, arranged from left to right for ranking, or set at horizontal distances from each other that reflect perceived differences. The position of a button does not affect its relationship to the auditory stimulus it represents.

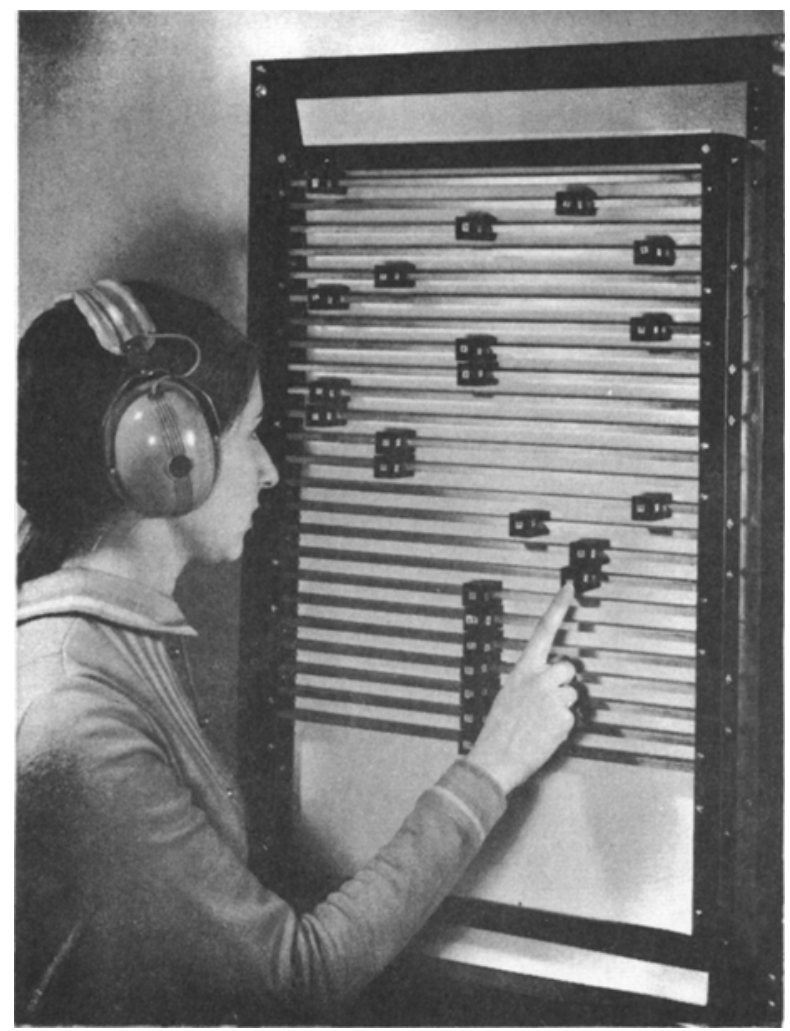

Fig. 1. View of the auditory sorting apparatus in use.
The display board (see Fig. 1) is an array of pushbuttons that slide on horizontal bars. Lateral pressure on a button causes it to move left or right along its bar, while pressure on a button in a direction normal to the plane of the display causes the button and its bar to move in that direction. Switch operation is accomplished by microswitches mounted on the frame as shown in Fig. 2, where the cover plate has been cut away to show the microswitch behind the near (right) end of each bar; another microswitch is out of sight behind the far (left) end. At least one of the two switches behind each bar is operated when the pushbutton moves inward no more than $1 / 32$ in., regardless of the position of the button on the bar. The microswitches are wired in parallel to the stimulus-control circuits so that operation of either one or both initiates a presentation of the stimulus assigned to that bar. Distributing the switching function in this way means that no electrical connections need be made to the sliding button itself. The springs of the microswitches provide the force that restores the bar to its original position when the pushbutton is released, and the cover plate limits the return movement of the bar.

The bars, made of $1 / 4 \times 1 / 2-$ in. aluminum, rest in slightly oversized notches in the phenol fiber supporting frame. The buttons, of $3 / 4$-in. sq phenol fiber, protrude $3 / 2$ in. in front of the bars. The U-shaped slot in the button extends beyond the bar to accommodate a brace at its rear. The $19 \times 24$-in. display board in Fig. 1 holds 30 bars, although any number of bars up to 30 may be used; those not needed in a particular experiment are easily removable.

The apparatus is adaptable to a variety of external circuits and therefore to many experimental conditions. In the simplest arrangement, the two microswitches on each bar are connected in parallel to a multicontact slave relay that presents a unique pattern of control voltages to the stimulus generation and control equipment (see Fig. 3). These voltages determine the signal parameters and one of them may trigger a gate that initiates the stimulus presentation. The stimulus gate can either run a preset

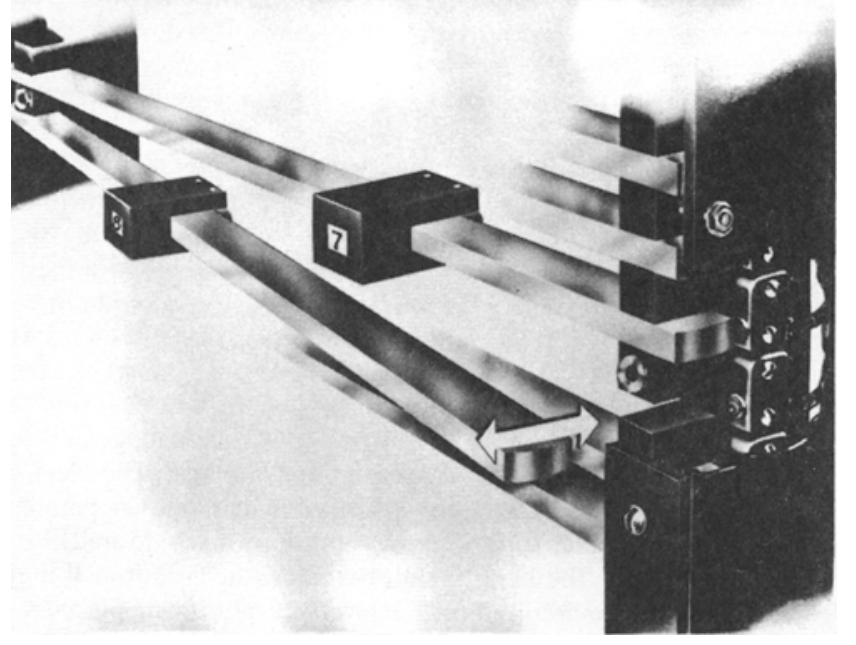

Fig. 2. Detail of construction showing placement of microswitch behind sliding bar. 
time course or remain open only while the button is depressed. In one experiment on similarity scaling of frequency-modulated tones, the stimulus generation and control equipment consisted of two voltage-controlled generators, voltage dividers, and an electronic switch. For another experiment on voice similarity, the apparatus will be connected to a small computer with a rapid random-access disk. Monosyllabic utterances, stored in digital form on the disk, will be retrieved and acoustically reproduced at the touch of a button.

At present, E must visually observe and record the configuration of buttons at the end of each trial. The recording function could be rendered automatic by adding a slide wire potentiometer to the back of each bar and connecting these potentiometers to voltage sources and measuring equipment. This equipment would record a button's position at each depression as well as the durations and sequence of button pushings (i.e., stimulus presentations). In this last respect, auditory stimulus sorting can furnish more potentially useful information than the sorting of manipulable visual stimuli, which does not yield precise information about S's experimental exposure to each stimulus.

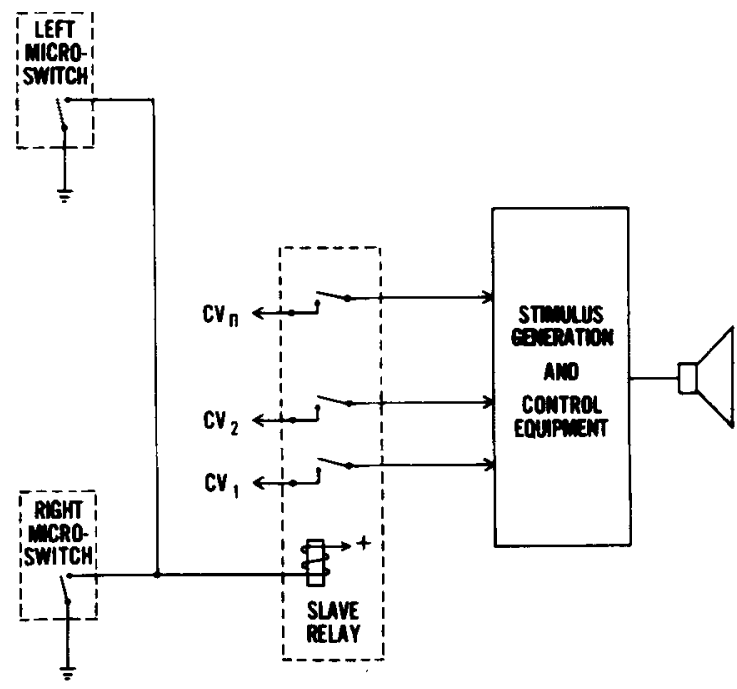

Fig. 3. Block diagram showing connection of sorting apparatus to stimulus generator.

\section{Simultaneous switching of auditory transients for two-channel presentation'}

\author{
RICHARD T. TOMSIC and LLOYD F. ELFNER, FLORIDA \\ STATE UNIVERSITY, Tallahassee, Fla. 32306
}

A modification of the Grason-Stadler Series $829 D \& E$ Electronic Switches that enables simultaneous cross-channel presentation of auditory signals at the two ears is described.

The human auditory system is extremely sensitive to interaural differences in arrival time of signals. A dichotically presented signal having an interaural arrival time discrepancy on the order of microseconds, is discriminable from a simultaneously presented signal. Therefore, it is imperative to exercise precise control of the onset and offset times. One of the most commonly used instruments employed in this area is the Grason-Stadler Series 829 (D or E) Electronic Switch. Even though the two channels of the switch are regulated by the same signal, slight differences in electronic tube characteristics can cause one channel to start conducting before the other. When two channels of the switch are used to turn on and turn off auditory signals simultaneously (within certain limits) at the two ears, the difference in the onset of the auditory signals is detectable.

A major portion of the difference in onset and offset times of the switched signal can be eliminated by matching the $6 \mathrm{SN} 7$ modulator tubes between the two channels (V51, V71, V52, and V72). Since tubes within each channel should be matched to minimize switching transients the task becomes one of matching four tubes. A potentiometer is supplied within each channel to compensate for tube differences to minimize switching transients but the same is not true for cross-channel matching.

In the unmodified circuit (Fig. 1a) that connects the biasing voltage or switching signal to the modulator tubes, the signal is passed through two $10 \mathrm{~K}$ resistors (R 112 and R 113, in the A switching circuits), one to each channel. In the modified circuit
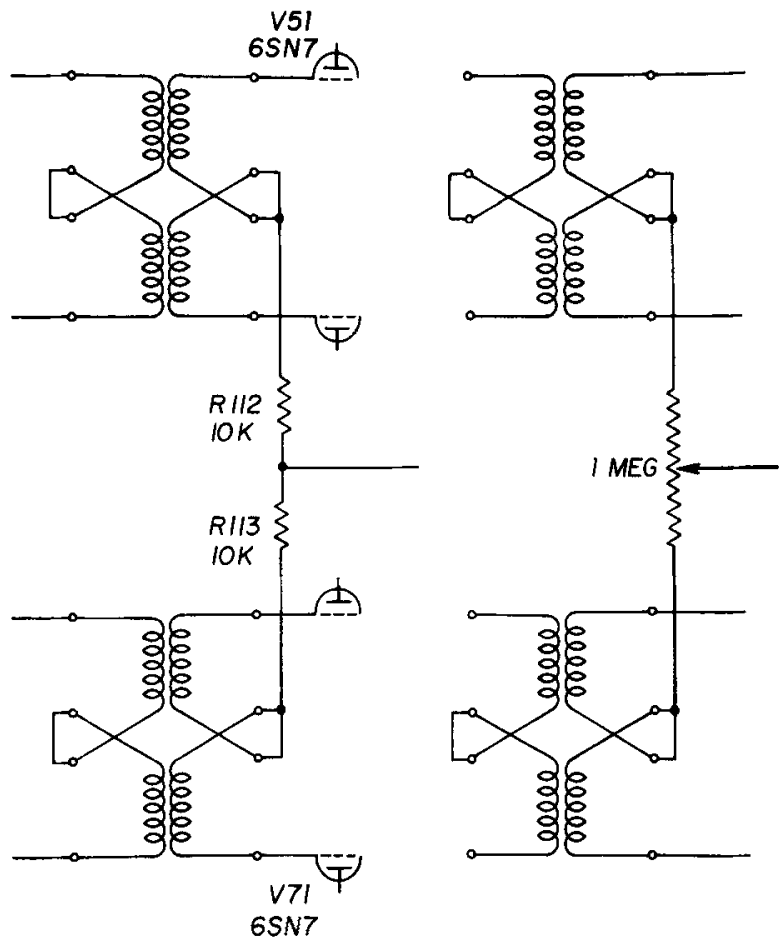

o. UNMODIFIED CIRCUIT

b. MODIFIED CIRCUIT 